Article

\title{
Perception Regarding Knowledge of COVID-19 Prevention in a Sample of a Middle Eastern and North African (MENA) Community in Houston, Texas, USA
}

\author{
Jenna Zamil ${ }^{1}$, Fatin Atrooz ${ }^{2}$, Zahra Majd ${ }^{3}$, Sarah Zeidat ${ }^{2}$, Ghalya Alrousan ${ }^{2}$, Susan Abughosh ${ }^{3}$
} and Samina Salim ${ }^{2, *}$

Citation: Zamil, J.; Atrooz, F.; Majd, Z.; Zeidat, S.; Alrousan, G.;

Abughosh, S.; Salim, S. Perception Regarding Knowledge of COVID-19 Prevention in a Sample of a Middle Eastern and North African (MENA) Community in Houston, Texas, USA. Int. J. Environ. Res. Public Health 2022, 19, 524. https://doi.org/10.3390/ ijerph19010524

Academic Editors: Nicola Luigi Bragazzi and Christian Napoli

Received: 18 November 2021

Accepted: 29 December 2021

Published: 4 January 2022

Publisher's Note: MDPI stays neutral with regard to jurisdictional claims in published maps and institutional affiliations.

Copyright: (C) 2022 by the authors. Licensee MDPI, Basel, Switzerland. This article is an open access article distributed under the terms and conditions of the Creative Commons Attribution (CC BY) license (https:// creativecommons.org/licenses/by/ $4.0 /)$.
1 College of Natural Sciences and Mathematics, University of Houston, Houston, TX 77204, USA; jennazamil@gmail.com

2 Department of Pharmacological and Pharmaceutical Science, College of Pharmacy, University of Houston, Houston, TX 77204, USA; fyatrooz@uh.edu (F.A.); sarazeidat@gmail.com (S.Z.); galrousa@central.uh.edu (G.A.)

3 Pharmaceutical Health Outcomes and Policy, College of Pharmacy, University of Houston, Houston, TX 77204, USA; majd.zahra@gmail.com (Z.M.); smabugho@central.uh.edu (S.A.)

* Correspondence: ssalim2@central.uh.edu; Tel.: +1-713-743-1776

\begin{abstract}
Background: Knowledge of COVID-19 prevention among communities is the first step towards protective behaviors. The objective of this study was to assess COVID-19 prevention knowledge among a Middle Eastern and North African community in Houston, Texas. (2) Methods: A cross-sectional study was conducted using a validated quantitative survey; survey questions consisted of three parts: COVID-19 specific questions, general health questions, and sociodemographic questions. A multivariable logistic regression model was used to determine predictors of perception of knowledge on preventing COVID-19 spread. The outcome of interest comprised of "good/excellent" versus "average and below" knowledge. (3) Results: A total of 366 participants (66.39\% males) completed the survey. A univariate analysis demonstrated significant differences in self-reported COVID-19 prevention knowledge among those with and without health insurance, different ages, level of knowledge, and perceived severity of COVID-19 infection. In the multivariate logistic regression, two predictors were identified: those in the 18-25-year-old group were more likely to have "excellent/good" knowledge on COVID-19 spread compared to the $\geq 40$-year-old group (OR: 6.36; 95\% CI: 1.38, 29.34). Those who somewhat agree with knowing how to protect themselves from COVID-19 were more likely to have "excellent/good" knowledge of preventing COVID-19 spread compared to those that neither agree nor disagree or disagree (OR: 7.74; 95\% CI: 2.58, 23.26). (4) Conclusions: Younger adults reported higher knowledge of COVID-19 prevention.
\end{abstract}

Keywords: Syrian refugees; stress; trauma; displacement; refugee mental health

\section{Introduction}

The catastrophic effects of the coronavirus disease 2019 (COVID-19), the highly contagious infectious disease caused by severe acute respiratory syndrome coronavirus 2 (SARS-CoV-2), has resulted in more than 5.0 million deaths worldwide, emerging as the most consequential global health crisis since the influenza pandemic of 1918 (WHO, November 2021 [1]). The COVID-19 disease is characterized by rapid transmission, fostered by close contact with an infected person [2,3]. Despite the unprecedented national measures in containing the outbreak, the success of prevention efforts is largely dependent on public behavior. Public adherence to preventive measures established by the Centers for Disease Control and Prevention (CDC) is critical in curbing the spread of the disease. Public adherence is influenced by knowledge and attitudes towards COVID-19. More than 40 million people living in the United States (US) are foreign-born including the refugee, 
immigrant, and migrant (RIM) populations who have settled in the US for a variety of different reasons [4,5]. The RIM group, critical for America's social, cultural, and economic vitality, is a heterogenous mixture of different ethnicities, cultures, and languages, thus representing a broad range of socioeconomic statuses, educational levels, occupations, and English proficiency levels [6], consequently, resulting in considerable variations in awareness, attitudes, and practices toward COVID-19 [7].

In this study, we have focused on immigrants from the Middle East and North Africa (MENA) region residing in Houston, Texas. The MENA community is a historically understudied group in the US, partly due to the lack of a well-defined taxonomic category in the US Census. Individuals of MENA descent are classified as "Caucasian" or "White" in the US Census [8]. Consequently, health behavior research data of the MENA group is mixed with Caucasian data, thus masking MENA-specific public health behaviors. Only a handful of studies have examined MENA health behaviors [9-12], emphasizing the need to conduct more systematic studies in this important group. The estimated number of MENA individuals in the US is more than 3.5 million [13], and Texas has the fourth largest MENA population in the country, with over 281,000 MENA Texas residents [14,15]. Houston reportedly has 98,300 MENA residents (Houston Chronicle [16]). According to the Arab American Institute, the largest population of MENA Texans reside in Harris and Fort Bend counties, including the Katy and Sugar Land area (The Texas Tribune, 2020 [17]). The present COVID-19 survey, therefore, targeted MENA individuals residing in the Harris and Fort Bend Counties in the Houston Metro area.

To encourage protective measures, knowledge on COVID-19 is critical. Several studies have pointed out a close relationship between sociodemographic characteristics and COVID-19 knowledge and vaccination status [18]. Among Middle Easterners, prior perceptions, location, and education level showed a possible connection with COVID-19 knowledge. For example, among medical students in Iran, COVID-19 knowledge was found to be accurate, with $79.6 \%$ of participants demonstrating high COVID-19 knowledge and over $90 \%$ demonstrating high levels of protective behavior [19]. However, a study evaluating COVID-19 knowledge among MENA residents in Dearborn, Michigan, found that the United States death toll of COVID-19 was "severely underestimated" by study participants, who predicted over 400,000 fewer deaths nationally than currently recorded [12]. These results suggest a need to evaluate existing knowledge and develop interventions to increase COVID-19 knowledge among Middle Eastern Americans. In Houston, the subpopulations of MENA immigrants with the lowest COVID-19 prevention knowledge levels have not yet been identified, which informs our research. Understanding perceptions and knowledge regarding COVID-19 and how to protect against infection among Middle Eastern Americans is the first step to designing effective interventions to promote vaccination as well as other behaviors, such as distancing, that can curb the virus in the community. Therefore, the objective of this study was to evaluate perceptions of knowledge on how to protect against COVID-19 among the MENA group and to identify subpopulations within this immigrant community who would benefit the most from educational outreach programs and vaccine promotion measures.

\section{Materials and Methods}

This is a cross-sectional survey-based study conducted between July and August 2021 among MENA members residing in the Houston area. All methods used and survey questionnaires utilized were approved by the Institutional Review Board (IRB, \#STUDY00003078) Committee for the Protection of Human Subjects, University of Houston, Houston, TX, USA.

Study participants: The present study was conducted during the COVID-19 pandemic; therefore, all institutional guidance and restrictions were followed; all communications were over the phone, text messaging, or online platforms (Zoom, TEAMS). After our study protocol was approved by the UH-IRB Committee, an anonymous, digital bilingual (English and Arabic) survey was created using a generalized survey service known as RedCap, and the survey link was sent to the MENA community through Houston-based 
501 (C) (3) non-profit organization MultiCultural Center (MCC), Webster, TX (http:// www.multiculturalcenter.net/, accessed on 27 July 2021). Date of survey dissemination 07/27/2021. MCC works with the MENA community in the Houston area. The RedCap survey dissemination was facilitated by MCC coordinator Dr. Uzma Khan (PharmD) by sharing the survey link to the MCC listserv. A study fellow, students, and research assistant also recruited participants using their social media pages. Anyone who was willing to participate in the survey and identified themselves as a Houston resident and belonged to the MENA community was included in the survey. Participants were included in the study by stating their country of origin from the Middle East and North Africa. The survey was distributed through a community organization listserv MCC, which serves the MENA community.

Inclusion Criteria: MENA adult men and women (country of origin: Algeria, Bahrain, Egypt, Iran, Iraq, Israel-Palestine, Jordan, Kuwait, Lebanon, Libya, Morocco, Oman, Qatar, Saudi Arabia, Syria, Tunisia, United Arab Emirates, and Yemen), 18 years old and above, who are settled in the Houston area were included in this study. Exclusion criteria: MENA individuals under the age of 18. MENA individuals who are residing out of the Houston area and non-members of the MENA group.

Survey: The survey was created by Dr. Nadia Abuelezam [11,20], William F. Connell School of Nursing, Boston College, MA. The survey comprised of previously validated questions that were originally written in English and translated into Arabic by a bilingual Arabic translator. The first page of the survey included the consent form, which has comprehensive information about the study and the principal investigator's contact information. In the consent form, participants were asked to provide consent for participation in the study. The quantitative survey questions consisted of three parts: COVID-19 specific questions, general health questions, and sociodemographic questions. COVID-19-related factors included: prior COVID-19 infection, perceived infection risk, perceived infection severity, perceived knowledge regarding self-protection from the COVID-19 infection, and perceived ability to avoid the infection. Health questions included: health insurance status, overall health status, smoking status, alcohol consumption, and disease comorbidities such as hypertension, hypercholesterolemia, obesity, diabetes, anxiety, and depression. The sociodemographic questions included: age, gender, race/ethnicity, country of origin, religion, immigration status, marital status, educational level, living status, and annual household income.

Statistical analysis: A descriptive analysis was performed to summarize participants' sociodemographic, general health, as well as responses to COVID-19-related questions. The frequencies for all categorical variables were calculated and described as percentages. Continuous variables were reported in mean \pm standard deviation (SD). Group differences were assessed using chi-square tests. A multivariable logistic regression model was conducted to determine predictors of perception of knowledge on preventing COVID-19 spread. The outcome of interest was having "good/excellent" versus "average and below" knowledge. Covariates included in the model were gender, age, education, marital status, living status, annual house income, religion, smoking status, alcohol consumption, health insurance, overall health perception, and comorbidities such as hypertension, hypercholesterolemia, obesity, and anxiety. COVID-19-related factors included prior infection, perceived infection risk, perceived infection severity, perceived knowledge of self-protection from the COVID-19 infection, and perceived ability to avoid infection. All statistical analyses were performed using SAS version 9.4 (SAS Institute, Cary, NC, USA) at an a priori significance level of 0.05 .

Sample size calculation: The G-power 3.1 statistical software was used for sample size estimation [21]. The estimated number of the MENA population in the Houston area is 98,300 . Accordingly, a total of 242 subjects were needed to provide $80 \%$ power to detect significance with a 0.15 effect size for a chi-square analysis at a $0.05 \alpha$-level. Additionally, we calculated that a minimum sample of 308 patients would provide $80 \%$ power to detect a 1.5 odds ratio for a two-tailed analysis at $\alpha<0.05$ using a logistic regression. 


\section{Results}

\subsection{Demographic Characteristics}

Table 1 shows the demographic characteristics and social circumstances of the 366 survey respondents including 243 males (66.39\%) and 123 females (33.61\%). A majority of the respondents $(67.76 \%)$ were within the 26-39-year age group. Around one third $(27.60 \%)$ of the participants reported having some college education and $48.09 \%$ reported attainment of college degrees. Of the total respondents, $67.21 \%$ were married, $21.58 \%$ were single, and $11.20 \%$ were widowed or divorced. The average annual income for almost half of the respondents $(46.72 \%)$ was between $\$ 45,000-\$ 100,000$. More than half $(53.01 \%)$ of the respondents identified themselves as Muslims, $34.43 \%$ as Christians, and 12.57\% as Jewish. Of all the participants, 144 reported smoking cigarettes or hookah (39.34\%), and $115(31.42 \%)$ reported drinking alcoholic beverages. Concerning respondents' overall health, the majority (65.30\%) stated as being in very good/good health. Some of the respondents reported the following health issues: hypertension $(54 ; 15.17 \%)$, high cholesterol $(48 ; 13.56 \%)$, and obesity $(52 ; 14.94 \%)$. In total, $29.55 \%$ of the respondents, 104 individuals, reported having anxiety.

Table 1. Baseline demographic and characteristics of knowledge on preventing COVID-19 spread.

\begin{tabular}{|c|c|c|c|c|}
\hline & \multirow[b]{2}{*}{ Total (\%) } & \multicolumn{2}{|c|}{ Knowledge-Prevent COVID-19 Spread } & \multirow{2}{*}{$p$ Value } \\
\hline & & $\begin{array}{l}\text { Excellent/ } \\
\text { Good }\end{array}$ & $\begin{array}{c}\text { Average/Poor/ } \\
\text { Terrible }\end{array}$ & \\
\hline \multicolumn{5}{|c|}{ Variable } \\
\hline \multicolumn{5}{|c|}{ Gender } \\
\hline Males & $243(66.39)$ & $182(65.47)$ & $61(69.32)$ & \multirow{2}{*}{0.5051} \\
\hline Females & $123(33.61)$ & $96(34.53)$ & $27(30.68)$ & \\
\hline \multicolumn{5}{|c|}{ Age (years) } \\
\hline $18-25$ & $85(23.22)$ & $72(25.90)$ & 13 (14.77) & \multirow{3}{*}{$0.0377^{*}$} \\
\hline $26-39$ & $248(67.76)$ & $185(66.55)$ & $63(71.59)$ & \\
\hline$\geq 40$ & $33(9.02)$ & $21(7.55)$ & $12(13.64)$ & \\
\hline \multicolumn{5}{|c|}{ Education } \\
\hline High school or less & $50(13.59)$ & $37(13.31)$ & 13 (14.77) & \multirow{4}{*}{0.6250} \\
\hline $\begin{array}{l}\text { Some college or } \\
\text { associate degree }\end{array}$ & 101(27.45) & $74(26.62)$ & $27(30.68)$ & \\
\hline College degree & $176(48.09)$ & $139(50.00)$ & $37(42.05)$ & \\
\hline Graduate degree & $39(10.66)$ & $28(10.07)$ & $11(12.50)$ & \\
\hline \multicolumn{5}{|c|}{ Marital Status } \\
\hline Married & $246(67.21)$ & $186(66.91)$ & $60(68.18)$ & \multirow{3}{*}{0.1329} \\
\hline Single & $79(21.59)$ & $65(23.38)$ & $14(15.91)$ & \\
\hline Divorced/Separated & $41(11.20)$ & $27(9.71)$ & $14(15.91)$ & \\
\hline \multicolumn{5}{|c|}{ Living Status } \\
\hline Own & $251(68.58)$ & $198(71.22)$ & $53(60.23)$ & \multirow{2}{*}{0.0528} \\
\hline Rent & 115 (31.42) & $80(28.78)$ & 35 (39.77) & \\
\hline
\end{tabular}


Table 1. Cont.

\begin{tabular}{|c|c|c|c|c|}
\hline & \multirow[b]{2}{*}{ Total (\%) } & \multicolumn{2}{|c|}{ Knowledge-Prevent COVID-19 Spread } & \multirow[b]{2}{*}{$p$ Value } \\
\hline & & $\begin{array}{l}\text { Excellent/ } \\
\text { Good }\end{array}$ & $\begin{array}{l}\text { Average/Poor/ } \\
\text { Terrible }\end{array}$ & \\
\hline \multicolumn{5}{|c|}{ Annual Household Income } \\
\hline$<\$ 25,000$ & $59(16.12)$ & $43(15.47)$ & $16(18.18)$ & \multirow{5}{*}{0.2790} \\
\hline $\begin{array}{l}\$ 25,000 \text { to } \\
<\$ 45,000\end{array}$ & $94(25.68)$ & $73(26.26)$ & $21(23.86)$ & \\
\hline $\begin{array}{l}\$ 45,000 \text { to } \\
<\$ 65,000\end{array}$ & $97(26.50)$ & $69(24.26)$ & $28(31.82)$ & \\
\hline $\begin{array}{l}\$ 65,000 \text { to } \\
<\$ 100,000\end{array}$ & $74(20.22)$ & $56(20.14)$ & $18(20.45)$ & \\
\hline$\geq \$ 100,000$ & $42(11.48)$ & $37(13.31)$ & $5(5.68)$ & \\
\hline \multicolumn{5}{|c|}{ Religion } \\
\hline Christian & $126(34.43)$ & $102(36.69)$ & $24(27.27)$ & \multirow{3}{*}{0.2676} \\
\hline Muslim & $194(53.01)$ & $142(51.08)$ & $52(59.09)$ & \\
\hline Jewish/other & $46(12.57)$ & $34(12.23)$ & $12(13.64)$ & \\
\hline \multicolumn{5}{|c|}{ Smoke } \\
\hline No & $222(60.66)$ & $161(57.91)$ & $61(69.32)$ & \multirow{2}{*}{0.0563} \\
\hline Yes & $144(39.34)$ & $117(42.09)$ & $27(30.68)$ & \\
\hline \multicolumn{5}{|c|}{ Drink Alcohol } \\
\hline No & $251(68.58)$ & $189(67.99)$ & $62(70.45)$ & \multirow{2}{*}{0.6637} \\
\hline Yes & $115(31.42)$ & $89(32.01)$ & $26(29.55)$ & \\
\hline \multicolumn{5}{|c|}{ Health Insurance } \\
\hline Yes & $291(79.51)$ & $228(82.01)$ & $63(71.59)$ & \multirow{2}{*}{0.0348 * } \\
\hline No & $75(20.49)$ & 50 (17.99) & $25(28.41)$ & \\
\hline \multicolumn{5}{|c|}{ Overall Health } \\
\hline Excellent & 105 (28.69) & $83(29.86)$ & $22(25.00)$ & \multirow{3}{*}{0.3036} \\
\hline Very Good/Good & $239(65.30)$ & $181(65.11)$ & $58965.91)$ & \\
\hline Fair/Poor & $22(6.01)$ & $14(5.04)$ & $899.09)$ & \\
\hline \multicolumn{5}{|c|}{ Hypertension } \\
\hline Yes & 54 (15.17) & $45(16.54)$ & $9(10.71)$ & \multirow{2}{*}{0.1929} \\
\hline No & $302(84.83)$ & $227(83.56)$ & 75 (89.29) & \\
\hline \multicolumn{5}{|c|}{ High Cholesterol } \\
\hline Yes & 48 (13.56) & $32(11.94)$ & $16(18.60)$ & \multirow{2}{*}{0.1163} \\
\hline No & $306(86.44)$ & $236(88.06)$ & $70(81.40)$ & \\
\hline \multicolumn{5}{|c|}{ Obesity } \\
\hline Yes & $52(14.94)$ & $41(15.36)$ & $11(13.58)$ & \multirow{2}{*}{0.6946} \\
\hline No & $296(85.06)$ & $226(84.64)$ & $70(86.42)$ & \\
\hline \multicolumn{5}{|c|}{ Anxiety } \\
\hline Yes & 104 (29.55) & $82(30.26)$ & $22(27.16)$ & \multirow{2}{*}{0.5918} \\
\hline No & $248(70.45)$ & $189(69.74)$ & $59(72.84)$ & \\
\hline
\end{tabular}


Table 1. Cont.

\begin{tabular}{|c|c|c|c|c|}
\hline & \multirow[b]{2}{*}{ Total (\%) } & \multicolumn{2}{|c|}{ Knowledge-Prevent COVID-19 Spread } & \multirow[b]{2}{*}{$p$ Value } \\
\hline & & $\begin{array}{l}\text { Excellent/ } \\
\text { Good }\end{array}$ & $\begin{array}{l}\text { Average/Poor/ } \\
\text { Terrible }\end{array}$ & \\
\hline \multicolumn{5}{|c|}{ Infected with COVID-19 } \\
\hline Yes & $69(18.96)$ & $52(18.71)$ & 17 (19.77) & \multirow{2}{*}{0.8261} \\
\hline No & $295(81.04)$ & $226(81.29)$ & $69(80.23)$ & \\
\hline \multicolumn{5}{|c|}{ COVID-19 Infection Risk } \\
\hline Extremely Likely & $41(11.23)$ & 31 (11.19) & $10(11.36)$ & \multirow[b]{3}{*}{0.7487} \\
\hline Somewhat Likely & $145(39.73)$ & $113(40.79)$ & $32(36.36)$ & \\
\hline $\begin{array}{c}\text { Neither Likely } \\
\text { nor Unlikely/ } \\
\text { Somewhat or } \\
\text { Extremely Unlikely }\end{array}$ & $179(49.04)$ & $133(48.01)$ & $46(52.27)$ & \\
\hline \multicolumn{5}{|c|}{ COVID-19 Infection Severity } \\
\hline Extremely Severe & $64(17.78)$ & $55(2015)$ & $9(10.34)$ & \multirow{4}{*}{0.0200 * } \\
\hline Somewhat Severe & $156(43.33)$ & $122(44.69)$ & $34(39.08)$ & \\
\hline $\begin{array}{l}\text { Neither Severe } \\
\text { nor Mild }\end{array}$ & $95(26.39)$ & $62(22.71)$ & $33(37.93)$ & \\
\hline $\begin{array}{l}\text { Somewhat Mild/ } \\
\text { Extremely Mild }\end{array}$ & $45(12.50)$ & $34(12.49)$ & $11(12.64)$ & \\
\hline \multicolumn{5}{|c|}{ Know Self-Protection from COVID-19 Infection } \\
\hline Strongly Agree & $123(33.61)$ & $107(38.49)$ & $16(18.18)$ & \multirow[b]{3}{*}{$0.0001^{* *}$} \\
\hline Somewhat Agree & $169(46.170$ & $138(49.64)$ & $31(35.23)$ & \\
\hline $\begin{array}{c}\text { Neither Agree } \\
\text { nor Disagree/ } \\
\text { Somewhat/Strongly } \\
\text { Disagree }\end{array}$ & $74(20.22)$ & 33 (11.87) & $41(46.59)$ & \\
\hline \multicolumn{5}{|c|}{ Avoiding COVID-19 Infection } \\
\hline $\begin{array}{c}\text { Extremely/ } \\
\text { Somewhat Easy }\end{array}$ & $200(55.250$ & $164(59.42)$ & $36(41.86)$ & \\
\hline $\begin{array}{l}\text { Neither Easy } \\
\text { nor Difficult }\end{array}$ & $111(30.66$ & $73(26.45)$ & 38 (44.19) & 0.0055 * \\
\hline $\begin{array}{l}\text { Somewhat/ } \\
\text { Extremely Difficult }\end{array}$ & $51(14.09)$ & 39 (14.13) & 12 (13.95) & \\
\hline
\end{tabular}

\subsection{COVID-19-Related Questions}

In this sample, 69 individuals (18.96\%) were infected with COVID-19. In parallel, 64 individuals $(17.78 \%)$ stated that the infection with COVID-19 was extremely severe. Only 41 individuals (11.23\%) declared a high likelihood of acquiring COVID-19 infection, and $14.09 \%$ reported that avoiding COVID-19 infection was extremely/somewhat difficult, $55.25 \%$ of respondents expressed avoiding the infection was extremely/somewhat easy, while 33.61\% reported having good knowledge of protection from COVID-19 infection.

Among all variables tested, the univariate analysis showed significant group differences between age groups in knowledge of how to prevent oneself from COVID-19 infection. Additionally, those with and without health insurance, level of knowledge about how to protect oneself against COVID-19, perceived difficulty of avoiding COVID-19 infection, and perceived COVID-19 infection severity also showed a significant difference in knowledge of COVID-19 infection prevention (Table 1). 


\subsection{Multivariable Logistic Regression Analysis}

In the multivariate logistic regression analysis, two predictors were identified: those in the age group 18-25 years old were more likely to have "excellent/good" knowledge on preventing COVID-19 spread compared to the age group of 40 years and above (OR: 6.36; 95\% CI: 1.38, 29.34). Those who somewhat agree with knowing how to protect themselves from COVID-19 were more likely to have "excellent/good" knowledge of preventing COVID-19 spread compared to those who neither agree nor disagree or disagree (OR: 7.74; 95\% CI: 2.58, 23.26) (Table 2).

Table 2. Multivariable logistic regression of knowledge on preventing COVID-19 spread.

\begin{tabular}{|c|c|c|}
\hline \multicolumn{3}{|c|}{ Excellent/Good Knowledge vs. Average/Poor/Terrible } \\
\hline & OR $(95 \% \mathrm{CI})$ & $p$ Value \\
\hline \multicolumn{3}{|c|}{ Variable } \\
\hline \multicolumn{3}{|c|}{ Gender } \\
\hline Male vs. Female & $0.75(0.37-1.54)$ & 0.4341 \\
\hline \multicolumn{3}{|c|}{ Age } \\
\hline $18-25$ vs. $\geq 40$ & $6.36(1.38-29.34)$ & 0.0223 * \\
\hline $26-39$ vs. $\geq 40$ & $2.99(0.86-10.40)$ & 0.6771 \\
\hline \multicolumn{3}{|c|}{ Education } \\
\hline$\leq$ High School vs. Graduate Degree & $1.89(0.40-8.94)$ & 0.745 \\
\hline Some College vs. Graduate Degree & $1.44(0.41-5.13)$ & 0.6813 \\
\hline College Degree vs. Graduate Degree & $2.63(0.81-8.53)$ & 0.1008 \\
\hline \multicolumn{3}{|c|}{ Marital Status } \\
\hline Married vs. Divorced/Separated & $2.60(0.92-7.39)$ & 0.1252 \\
\hline Single vs. Divorced/Separated & $1.94(0.60-6.32)$ & 0.6962 \\
\hline \multicolumn{3}{|c|}{ Living Status } \\
\hline Own vs. Rent & $0.95(0.44-2.08)$ & 0.9054 \\
\hline \multicolumn{3}{|c|}{ Annual Household Income } \\
\hline$<\$ 25,000$ vs. $\geq \$ 100,000$ & $0.08(0.01-0.47)$ & 0.1091 \\
\hline$\$ 25,000$ to $<\$ 45,000$ vs. $\geq \$ 100,000$ & $0.12(0.02-0.64)$ & 0.4358 \\
\hline$\$ 45,000$ to $<\$ 65,000$ vs. $\geq \$ 100,000$ & $0.12(0.02-0.57)$ & 0.3838 \\
\hline$\$ 65,000$ to $<\$ 100,000$ vs. $\geq \$ 100,000$ & $0.08(0.02-0.44)$ & 0.0985 \\
\hline \multicolumn{3}{|c|}{ Religion } \\
\hline Christian vs. Jewish/Other & $2.27(0.76-6.76)$ & 0.0978 \\
\hline Muslim vs. Jewish/Other & $1.30(0.47-3.60)$ & 0.6994 \\
\hline \multicolumn{3}{|c|}{ Smoke } \\
\hline No vs. Yes & $0.48(0.23-1.02)$ & 0.0573 \\
\hline \multicolumn{3}{|c|}{ Drink Alcohol } \\
\hline No vs. Yes & $1.43(0.66-3.11)$ & 0.3647 \\
\hline \multicolumn{3}{|c|}{ Health Insurance } \\
\hline Yes vs. No & $0.84(0.36-1.93)$ & 0.6775 \\
\hline \multicolumn{3}{|c|}{ Overall Health } \\
\hline Excellent vs. Fair/Poor & $1.78(0.40-7.94)$ & 0.9372 \\
\hline Very Good/Good vs. Fair/Poor & $2.94(0.74-11.73)$ & 0.0753 \\
\hline
\end{tabular}


Table 2. Cont.

\begin{tabular}{|c|c|c|}
\hline \multicolumn{3}{|c|}{ Excellent/Good Knowledge vs. Average/Poor/Terrible } \\
\hline & OR $(95 \%$ CI) & $p$ Value \\
\hline \multicolumn{3}{|c|}{ Hypertension } \\
\hline Yes vs. No & $3.06(0.92-10.23)$ & 0.0692 \\
\hline \multicolumn{3}{|c|}{ High Cholesterol } \\
\hline Yes vs. No & $0.83(0.26-2.71)$ & 0.7615 \\
\hline \multicolumn{3}{|l|}{ Obesity } \\
\hline Yes vs. No & $1.77(0.62-5.08)$ & 0.29 \\
\hline \multicolumn{3}{|l|}{ Anxiety } \\
\hline Yes vs. No & $1.17(0.53-2.59)$ & 0.6904 \\
\hline \multicolumn{3}{|c|}{ Infected with COVID-19 } \\
\hline Yes vs. No & $0.54(0.23-1.31)$ & 0.1725 \\
\hline \multicolumn{3}{|c|}{ COVID-19 Infection Risk } \\
\hline $\begin{array}{l}\text { Extremely Likely vs. Neither Likely nor } \\
\text { Unlikely/Somewhat Unlikely }\end{array}$ & $1.99(0.48-8.34)$ & 0.2509 \\
\hline $\begin{array}{l}\text { Somewhat Likely vs. Neither Likely nor } \\
\text { Unlikely/Somewhat Unlikely }\end{array}$ & $0.78(0.38-1.63)$ & 0.1908 \\
\hline \multicolumn{3}{|c|}{ COVID-19 Infection Severity } \\
\hline Extremely Severe vs. Somewhat/Extremely Mild & $1.88(0.47-7.45)$ & 0.2369 \\
\hline Somewhat Severe vs. Somewhat/Extremely Mild & $0.92(0.31-2.74)$ & 0.454 \\
\hline $\begin{array}{l}\text { Neither Severe nor Mild vs. } \\
\text { Somewhat/Extremely Mild }\end{array}$ & $0.98(0.32-2.99)$ & 0.6432 \\
\hline \multicolumn{3}{|c|}{ Know Self-Protection from COVID-19 Infection } \\
\hline Strongly Agree vs. Neither Agree nor Disagree/ & \multirow{2}{*}{$7.74(2.58-23.26)$} & \multirow{2}{*}{0.0043 * } \\
\hline Somewhat Disagree/Strongly Disagree & & \\
\hline Somewhat Agree vs. Neither Agree nor Disagree/ & \multirow{2}{*}{$4.49(1.84-10.96)$} & \multirow{2}{*}{0.177} \\
\hline Somewhat Disagree/Strongly Disagree & & \\
\hline \multicolumn{3}{|c|}{ Avoiding COVID-19 Infection } \\
\hline $\begin{array}{c}\text { Extremely Easy/Somewhat Easy vs. Somewhat } \\
\text { Difficult/Extremely Difficult }\end{array}$ & $1.02(0.39-2.98)$ & 0.3377 \\
\hline $\begin{array}{l}\text { Neither Easy nor Difficult vs. Somewhat } \\
\text { Difficult/Extremely Difficult }\end{array}$ & $0.49(0.17-1.42)$ & 0.0629 \\
\hline
\end{tabular}

\section{Discussion}

The present study assessed knowledge, perceptions, and practices related to COVID19 among Houston MENA residents. In this study, younger participants (18-25 years old) demonstrated more knowledge about COVID-19 transmission and prevention than the older age group, an observation which could be explained by the increased use of social media outlets by this group when compared to the older age group. These results are consistent with several previous studies conducted on COVID-19 knowledge, awareness, and attitudes in the MENA group residing outside of the US [22,23]. According to our study, the 18-25-year-old age group was more likely to report "good/excellent" COVID-19 prevention knowledge than other groups. Interestingly, this age group represents the largest age demographic in the study that is represented on the social media site TikTok, as nearly two-thirds of the site's user base is under the age of 29. As stated by Ostrovsky et al., the hashtag "coronavirus" generated 93.1 billion views, demonstrating the wide 
social media coverage of the pandemic [24]. Thus, coronavirus information access and its rapid assimilation through social media could lead to younger adults perceiving a higher knowledge level of COVID-19 prevention than older adult age groups.

Furthermore, young adults are believed to more likely respond to peer influence than older adults [25]. Thus, it is possible that knowledge of preventing COVID-19 among young adults is popularized due to peer influence, providing another factor that could inform our study's results. However, this rapid assimilation of information among young people also presents the problem of misinformation and raises the possibility of misrepresented information being distributed, allowing a higher perceived level of COVID-19 knowledge than truly gained. This informs the study's limitations on providing only a self-reported perception of COVID-19 prevention knowledge, as the accuracy of perceived knowledge on preventing COVID-19 was not evaluated. Future studies should focus on the source of young adults' COVID-19 information and evaluate actual knowledge of COVID-19 in comparison with our results. Additionally, the present study illustrates the need for designing a COVID-19 educational outreach strategy catered towards older adults in the MENA immigrant population, as this group reported lower perceived confidence in their knowledge about preventing COVID-19. Clearly, the healthcare needs of the elderly MENA community in general, and COVID-19 prevention strategies in particular, need attention. Surprisingly, only a handful of studies have examined the healthcare needs of older MENA Americans $[9,26,27]$. Thus, more work is needed in this area to understand how to best care for aging MENA Americans and to ensure effective and feasible COVID-19 prevention strategies for this group.

Findings from our study also suggest that individuals who reported relatively adequate knowledge on how to protect themselves from COVID-19 infection also reported higher levels of COVID-19 prevention knowledge. In a study by Riad Abanoub et al., knowledge about COVID-19 showed a positive correlation with protective behaviors against COVID-19 [28]. These results underscore the importance of adopting language-tailored culturally competent COVID-19 educational programs for MENA immigrants, as customized programs may lead to greater acceptance of COVID-19 vaccinations, higher knowledge, and improved practice of protective health behaviors. Such language-tailored interventions by incorporating cultural beliefs may also mitigate apprehensions about COVID-19. Constant monitoring of vaccination status, COVID-19 prevalence, the latest scientific evidence, and public opinion monitoring are needed for effective disease prevention [18].

\section{Conclusions}

Our results can help inform the direction of future educational and public health endeavors in Houston's MENA community towards populations in need, allowing MENA adults to increase their knowledge on protection against COVID-19 and thus, allow for apprehensions about vaccination and other protective measures to curb the spread of this virus within and beyond the MENA community. Finally, more attention is needed to better understand the social and health needs of the MENA group in the context of the existing racial and minority health landscape in the US. This is critical for better understanding of immigrant health in the US.

\section{Limitations}

Several limitations of this study can be reported. The cross-sectional design precludes cause-and-effect relationships. Findings of this study are based on self-reported data in a relatively small sample size and should be interpreted given these limitations. The MENA community is a heterogenous group including individuals of Arab, Turkish, and Persian descent, with varying socioeconomic status. However, our sample included predominantly Arab members; therefore, COVID-19 vaccination-related behaviors among the Turkish and Persian community were not discussed. Future studies are recommended to evaluate the knowledge of COVID-19 prevention among the MENA community using different study designs and larger samples. Finally, given the scarce literature in this population, this 
cross-sectional study served as a hypothesis-generating study using a validated survey of demographics and health-related and knowledge-based variables, but it was not built on a fully developed conceptual framework. Taking our findings into consideration, more conceptually based future work is warranted and is currently in progress.

Supplementary Materials: The following supporting information can be downloaded at: https: / /www.mdpi.com/article/10.3390/ijerph19010524/s1. Survey Questionnaire.

Author Contributions: F.A., G.A. and S.S. conducted the literature review and prepared and modified survey questionnaires shared by Nadia N. Abeulezam, William F. Connell School of Nursing, Boston College, MA. Analyses were conducted by Z.M., J.Z. and S.A.; J.Z., S.S. and F.A. wrote the first draft of the manuscript. S.S. finalized the draft after several layers of edits and iterations by all authors. All authors have read and agreed to the published version of the manuscript.

Funding: This work was supported by the Houston Global Health Collaborative Student Research and Service Grant awarded to the College of Pharmacy doctoral student Sarah Zeidat and Grants to Enhance Research on Racism, University of Houston, awarded to Samina Salim.

Institutional Review Board Statement: All communication forms and survey questionnaires utilized in the study were approved by the Institutional Review Board (IRB) Committee (STUDY00003078) for the Protection of Human Subjects, University of Houston (UH), Houston, TX, US. The study was conducted according to the guidelines of the Declaration of United States and approved by the Institutional Review Board (or Ethics Committee) of University of Houston (STUDY00003078, 8 July 2021). The Survey Questionnaire can be found at the supplementary materials.

Informed Consent Statement: Informed consent was obtained from all subjects involved in the study.

Data Availability Statement: The data presented in this study are available on request from the corresponding author.

Acknowledgments: We are grateful to Nadia N. Abeulezam (William F. Connell School of Nursing, Boston College, Boston, MA, USA) for sharing her survey questions and for her input, which have helped in conducting this study. The authors gratefully acknowledge the help and support of Multicultural Center (MCC) study coordinator Uzma Khan (PharmD) for her help in dissemination of the survey to MENA community members of MCC.

Conflicts of Interest: The authors declare that the research was conducted in the absence of any commercial or financial relationships that could be construed as a potential conflict of interest.

\section{References}

1. World Health Organization (WHO). Coronavirus (COVID-19) Dashboard. 2021. Available online: https://covid19.who.int/ (accessed on 20 December 2021).

2. Chan, J.F.W.; Yuan, S.; Kok, K.H.; To, K.K.W.; Chu, H.; Yang, J.; Xing, F.; Liu, J.; Yip, C.C.Y.; Poon, R.W.S.; et al. A familial cluster of pneumonia associated with the 2019 novel coronavirus indicating person-to-person transmission: A study of a family cluster. Lancet 2020, 395, 514-523. [CrossRef]

3. Huang, C.; Wang, Y.; Li, X.; Ren, L.; Zhao, J.; Hu, Y.; Zhang, L.; Fan, G.; Xu, J.; Gu, X.; et al. Clinical features of patients infected with 2019 novel coronavirus in Wuhan, China. Lancet 2020, 395, 497-506. [CrossRef]

4. Jonny, S. The Erasure of Middle Eastern and North African Immigrants from the American Narrative: A Case for Adding a Mena Category to the United States. Geo. Immigr. LJ 2020, 35, 1009.

5. Abbas, M.; Aloudat, T.; Bartolomei, J.; Carballo, M.; Durieux-Paillard, S.; Gabus, L.; Jablonka, A.; Jackson, Y.; Kaojaroen, K.; Koch, D.; et al. Migrant and refugee populations: A public health and policy perspective on a continuing global crisis. Antimicrob. Resist. Infect. Control. 2018, 7, 113. [CrossRef] [PubMed]

6. Juang, L.P.; Simpson, J.A.; Lee, R.M.; Rothman, A.J.; Titzmann, P.F.; Schachner, M.K.; Korn, L.; Heinemeier, D.; Betsch, C. Using attachment and relational perspectives to understand adaptation and resilience among immigrant and refugee youth. Am. Psychol. 2018, 73, 797. [CrossRef] [PubMed]

7. Thomas, C.M.; Osterholm, M.T.; Stauffer, W.M. Critical considerations for COVID-19 vaccination of refugees, immigrants, and migrants. Am. J. Trop. Med. Hyg. 2021, 104, 433. [CrossRef]

8. Lybarger, L.D. Whitewashed: America's Invisible Middle Eastern Minority. J. Am. Ethn. History 2012, 31, 154-156. [CrossRef]

9. Dallo, J.F.; Kindratt, T.B. Disparities in vaccinations and cancer screening among US-and foreign-born Arab and European American non-Hispanic White women. Women's Health Issues 2015, 25, 56-62. [CrossRef] 
10. Pruitt, C.N.; Reese, C.S.; Grossardt, B.R.; Shire, A.M.; Creedon, D.J. Completion of the human papillomavirus vaccination series lags in Somali adolescents. J. Low Genit. Tract Dis. 2013, 17, 280-288. [CrossRef]

11. Abouhala, S.; Hamidaddin, A.; Taye, M.; Glass, D.J.; Zanial, N.; Hammood, F.; Allouch, F.; Abuelezam, N.N. A National Survey Assessing COVID-19 Vaccine Hesitancy Among Arab Americans. J. Racial Ethn. Health Dispar. 2021, 1-9. [CrossRef]

12. Ismail, M.A.; Khalil, M.; Najdi, R.; Blackwood, R.A. The Assessment of COVID-19 Knowledge and Attitudes Among a Middle Eastern North African Community in Dearborn, Michigan. J. Commun. Health 2021, 1-7. [CrossRef]

13. Albqoor, M.A.; Chen, J.L.; Weiss, S.; Waters, C.; Choi, J. Self-rated health of middle eastern immigrants in the US: A national study. Public Health 2020, 180, 64-73. [CrossRef]

14. Awad, G.H.; Kia-Keating, M.; Amer, M.M. A model of cumulative racial-ethnic trauma among Americans of Middle Eastern and North African (MENA) descent. Am. Psychol. 2019, 74, 76. [CrossRef]

15. Tehranian, J. Whitewashed: America's Invisible Middle Eastern Minority; NYU Press: New York, NY, USA, 2008 ; Volume 46.

16. Houston Chronicle. Available online: https://www.houstonchronicle.com/news/houston-texas/houston/article/ArabAmericans-others-of-Middle-Eastern-descent-14075860.php (accessed on 20 December 2021).

17. The Texas Tribune. 2020. Available online: https://www.texastribune.org/2020/10/19/2020-census-texas-arab-mena. (accessed on 20 December 2021).

18. Gallè, F.; Sabella, E.A.; Roma, P.; De Giglio, O.; Caggiano, G.; Tafuri, S.; Da Molin, G.; Ferracuti, S.; Montagna, M.T.; Liguori, G.; et al. Knowledge and Acceptance of COVID-19 Vaccination among Undergraduate Students from Central and Southern Italy. Vaccines 2021, 9, 638. [CrossRef] [PubMed]

19. Taghrir, H.M.; Borazjani, R.; Shiraly, R. COVID-19 and Iranian medical students; a survey on their related-knowledge, preventive behaviors and risk perception. Arch. Iran. Med. 2020, 23, 249-254. [CrossRef] [PubMed]

20. Allen, J.D.; Abuelezam, N.N.; Rose, R.; Fontenot, H.B. Factors associated with the intention to obtain a COVID-19 vaccine among a racially/ethnically diverse sample of women in the USA. Transl. Behav. Med. 2021, 11, 785-792. [CrossRef] [PubMed]

21. Faul, F.; Erdfelder, E.; Lang, A.G.; Buchner, A. G*Power 3: A flexible statistical power analysis program for the social, behavioral, and biomedical sciences. Behav. Res. Methods 2007, 39, 175-191. [CrossRef] [PubMed]

22. Alahdal, H.; Basingab, F.; Alotaibi, R. An analytical study on the awareness, attitude and practice during the COVID-19 pandemic in Riyadh, Saudi Arabia. J. Infect. Public Health 2020, 13, 1446-1452. [CrossRef] [PubMed]

23. Qutob, N.; Awartani, F. Knowledge, attitudes and practices (KAP) towards COVID-19 among Palestinians during the COVID-19 outbreak: A cross-sectional survey. PLoS ONE 2021, 16, e0244925. [CrossRef]

24. Ostrovsky, A.M.; Chen, J.R. TikTok and its role in COVID-19 information propagation. J. Adolesc. Health 2020, 67, 730. [CrossRef]

25. Andrews, J.L.; Foulkes, L.; Blakemore, S.-J. Peer influence in adolescence: Public-health implications for COVID-19. Trends Cogn. Sci. 2020, 24, 585-587. [CrossRef] [PubMed]

26. Ajrouch, K.J. Resources and Well-being Among Arab-American Elders. J. Cross Cult. Gerontol. 2007, 22, 183-184. [CrossRef] [PubMed]

27. Abuelezam, N.N.; El-Sayed, A.M.; Galea, S. The health of Arab Americans in the United States: An updated comprehensive literature review. Front. Public Health 2018, 6, 262. [CrossRef] [PubMed]

28. Riad, A.; Huang, Y.; Zheng, L.; Elavsky, S. COVID-19 Induced Anxiety and Protective Behaviors during COVID-19 Outbreak: Scale Development and Validation. Available online: https:/ /www.medrxiv.org/content/10.1101/2020.05.05.20050419v1 (accessed on 20 December 2021). 